\title{
Identification of hub genes and small-molecule compounds related to intracerebral hemorrhage with bioinformatics analysis
}

\author{
Zhendong Liu ${ }^{\text {Equal first author, } 1,2}$, Ruotian Zhang Equal first author, 1,2 , Xin Chen ${ }^{1,2}$, Penglei Yao ${ }^{1,2}$, Tao Yan ${ }^{1,2}$, Wenwu Liu ${ }^{1,2}$, \\ Jiawei Yao $^{1,2}$, Sokhatskii Andrei $^{3}$, Gareev F. Ilgiz ${ }^{3}$, Shiguang Zhao ${ }^{\text {Corresp. } 1,2}$ \\ ${ }^{1}$ the First Affiliated Hospital of Harbin Medical University, Department of Neurosurgery, Harbin, Heilongjiang Province, People's Republic of China \\ 2 Harbin Medical University, Institute of Brain Science, Harbin, Heilongjiang Province, People's Republic of China \\ 3 Baskir State Medical University, Ufa, Russia \\ Corresponding Author: Shiguang Zhao \\ Email address: guangsz@hotmail.com
}

Background.Because of the complex mechanisms of injury, conventional surgical treatment and early blood pressure control does not significantly reduce mortality or improve patient prognosis in cases of intracerebral hemorrhage $(\mathrm{ICH})$. We aimed to identify the hub genes associated with intracerebral hemorrhage, to act as therapeutic targets, and to identify potential small-molecule compounds for treating $\mathrm{ICH}$.

Methods. The GSE24265 dataset, consisting of data from 4 perihematomal brain tissues and 7 contralateral brain tissues, was downloaded from the Gene Expression Omnibus (GEO) database and screened for differentially expressed genes (DEGs) in $\mathrm{ICH}$, with a fold change (FC) value of (|log2FC|) > 2 and a P-value of $<0.05$ set as cut-offs. The functional annotation of DEGs was performed using Gene Ontology (GO) resources, and the cell signaling pathway analysis of DEGs was performed using the Kyoto Encyclopedia of Genes and Genomes (KEGG), with a P-value of $<0.05$ set as the cut-off. We constructed a protein-protein interaction (PPI) network to clarify the interrelationships between the different DEGs and to select the hub genes with significant interactions. Next, the DEGs were analyzed using the CMap tool to identify small-molecule compounds with potential therapeutic effects. Finally, we verified the expression levels of the hub genes by RT-qPCR on the rat ICH model.

Result. A total of 59 up-regulated genes and 8 down-regulated genes associated with ICH were identified. The biological functions of DEGs associated with ICH are mainly involved in the inflammatory response, chemokine activity, and immune response. The KEGG analysis identified several pathways significantly associated with ICH, including but not limited to HIF-1, TNF, toll-like receptor, cytokinecytokine receptor interaction, and chemokine molecules. A PPI network consisting of 57 nodes and 373 edges was constructed using STRING, and 10 hub genes were identified with Cytoscape software. These hub genes are closely related to secondary brain injury induced by ICH. RT-qPCR results showed that the expression of ten hub genes was significantly increased in the rat model of ICH. In addition, a CMap analysis of three small-molecule compounds revealed their therapeutic potential.

Conclusion. In this study we obtained ten hub genes, such as IL6, TLR2, CXCL1, TIMP1, PLAUR, SERPINE1, SELE, CCL4, CCL20, and CD163, which play an important role in the pathology of ICH. At the same time, the ten hub genes obtained through PPI network analysis were verified in the rat model of $\mathrm{ICH}$. In addition, we obtained three small molecule compounds that will have therapeutic effects on ICH, including Hecogenin, Lidocaine, and NU-1025.

Peer) reviewing PDF | (2019:04:36749:2:0:NEW 16 Aug 2019) 


\section{Identification of hub genes and small-molecule compounds}

\section{2 related to intracerebral hemorrhage with bioinformatic}

\section{3 analysis}

4 Zhendong Liu ${ }^{1,2}$, Ruotian Zhang ${ }^{1,2}$, Xin Chen ${ }^{1,2}$, Penglei Yao ${ }^{1,2}$, Tao Yan ${ }^{1,2}$, Wenwu Liu ${ }^{1,2}$,

5 Jiawei Yao $^{1,2}$, Sokhatskii Andrei ${ }^{3}$, Gareev F. Ilgiz ${ }^{3}$, Shiguang Zhao ${ }^{1,2 *}$

$6{ }^{1}$ Department of Neurosurgery, The First Affiliated Hospital of Harbin Medical University, Harbi

7 n, Heilongjiang Province, People's Republic of China

8 'Institute of Brain Science, Harbin Medical University, Harbin, Heilongjiang Province, People's

9 Republic of China

10 3askir State Medical University, Ufa, Russia

11

12

13

14

15

*Zhendong Liu ${ }^{1,2}$ and Ruotian Zhang ${ }^{1,2}$ contributed equally to this work.

Corresponding Author:

Shiguang Zhao

No. 23 Youzheng Street, Harbin, Heilongjiang Province, 150001, People's Republic of China Email address: guangsz@hotmail.com 
39 Abstract

40 Background. Because of the complex mechanisms of injury, conventional surgical treatment 41 and early blood pressure control does not significantly reduce mortality or improve patient 42 prognosis in cases of intracerebral hemorrhage (ICH). We aimed to identify the hub genes 43 associated with intracerebral hemorrhage, to act as therapeutic targets, and to identify potential 44 small-molecule compounds for treating ICH.

45 Methods. The GSE24265 dataset, consisting of data from 4 perihematomal brain tissues and 7 46 contralateral brain tissues, was downloaded from the Gene Expression Omnibus (GEO) database and screened for differentially expressed genes (DEGs) in ICH, with a fold change (FC) value of $(|\log 2 \mathrm{FC}|)>2$ and a P-value of $<0.05$ set as cut-offs. The functional annotation of DEGs was performed using Gene Ontology (GO), and the cell signaling pathway analysis of DEGs was performed using the Kyoto Encyclopedia of Genes and Genomes (KEGG), with a P-value of $<$ 0.05 set as the cut-off. We constructed a protein-protein interaction (PPI) network to clarify the interrelationships between the different DEGs and to select the hub genes with significant interactions. Next, the DEGs were analyzed using the CMap tool to identify small-molecule compounds with potential therapeutic effects. Finally, we verified the expression levels of the hub genes by RT-qPCR on the rat ICH model. identified. The biological functions of DEGs associated with $\mathrm{ICH}$ are mainly involved in the inflammatory response, chemokine activity, and immune response. The KEGG analysis identified several pathways significantly associated with ICH, including but not limited to HIF-1, TNF, toll-like receptor, cytokine-cytokine receptor interaction, and chemokine. A PPI network consisting of 57 nodes and 373 edges was constructed using STRING, and 10 hub genes were identified with Cytoscape software. These hub genes are closely related to secondary brain injury induced by ICH. RT-qPCR results showed that the expression of ten hub genes was significantly increased in the rat model of ICH. In addition, a CMap analysis of three small-molecule compounds revealed their therapeutic potential.

66 Conclusion. In this study we obtained ten hub genes, such as IL6, TLR2, CXCL1, TIMP1, PLAUR, SERPINE1, SELE, CCL4, CCL20, and CD163, which play an important role in the pathology of ICH. At the same time, the ten hub genes obtained through PPI network analysis 
69

70

71

\section{Introduction}

73

74

75

76

77

78

79

80

81

82

83

84

85

86

87

88

89

90

91

92

93

94

95

96

97

98

were verified in the rat model of ICH. In addition, we obtained three small molecule compounds that will have therapeutic effects on ICH, including Hecogenin, Lidocaine, and NU-1025.

On account of its high morbidity and mortality, intracerebral hemorrhage (ICH) is a worldwide public health concern (Qureshi et al. 2001). About 2 million people experience ICH every year, and its incidence is still rising (Labovitz et al. 2005; Qureshi et al. 2007). Half of patients die within a year, and two-third of the survivors become permanently disabled (Fogelholm et al. 2005). The poor prognosis of patients with ICH is mainly related to its complex pathogenesis. The pathological process of ICH can be divided into primary injury, which consists of mechanical compression caused by hematoma, and secondary injury, which is caused by various factors such as cytotoxicity of blood, hypermetabolism, excitotoxicity, oxidative stress and inflammation (Aronowski \& Zhao 2011). Several studies have shown that treatments targeting the ICH-induced cellular processes and their downstream molecules may be effective (Morioka \& Orito 2017).

According to previous reports, the cytokines released by various inflammatory cells such as leukocytes, macrophages, and microglia after the onset of ICH were involved in the formation of secondary brain injury caused by ICH (Wang \& Dore 2007). For example, microglia/macrophages activated after the ICH event can cause an increase in the expression levels of TNF-a and IL-1 $\beta$, which can aggravate brain damage (Aronowski \& Hall 2005; Wagner 2007). In the ICH model constructed by animals, the expression of TNF- $\alpha$ was significantly increased, which promoted the formation of cerebral edema (Hua et al. 2006). Toll-like receptors (TLRs) play an irreplaceable role in the regulation of immune responses and inflammatory responses (Akira \& Takeda 2004; Takeda \& Akira 2004). TLRs are a family of transmembrane proteins, and it has been reported in the literature that the significant increase in expression levels of TLR2 and TLR4 in peripheral monocytes of ICH patients is significantly associated with poor prognosis in patients (Rodriguez-Yanez et al. 2012). In addition, the increased expression levels of proinflammatory genes such as CD36, iNOS, and MMP-9 all aggravated the pathological progression of ICH (Zhao et al. 2009; Zhao et al. 2007). Therefore, we have reason to speculate that there should be many potential genes in the pathological process of ICH that may play 
99 important regulatory roles. However, the expression profile and function of mRNAs in the course of 100 ICH have not yet been fully explained.

101 Presently, research on ICH treatment has mostly focused on the clinical aspects of 102 therapeutic interventions, and investigations into the molecular mechanisms underlying the 103 pathology of ICH remain scarce (Yin et al. 2017). Therefore, identifying the therapeutic targets 104 for ICH at the molecular level may improve the existing treatment strategies for ICH. Although 105 previous reports by Russell et al on the expression profile of mRNAs have revealed the differentially

106

107

108

109

110

111

112

113

114

115

116

117

118

119

120

121

122

123

124

125

126

127 expressed genes (DEGs) in the pathology of $\mathrm{ICH}$, the main focus of the study was on the top ten upregulated genes and ten down-regulated genes with the difference of the largest fold change. However, their research ignores the interaction between DEGs, which is crucial to understanding the biological significance of DEGs.

Our current research goal is to explore the expression profiles of mRNAs in ICH patients to screen for hub genes through the PPI network and subsequently analyze their functions. Furthermore, the rat model of ICH has been established to verify the expression level of hub genes by RT-qPCR. In addition, the DEGs were analyzed using the CMap tool to identify smallmolecule compounds with potential therapeutic effects on ICH.

\section{Materials \& Methods}

Data collection

The gene expression profiles of patients with ICH were obtained from the GEO database (https://www.ncbi.nlm.nih.gov/geo/) (Clough \& Barrett 2016), a public database for researchers. GSE24265, which includes the data from four spontaneous cases of intracerebral hemorrhage, relied on the Agilent GPL570 platform (Affymetrix Human Genome U133 Plus 2.0 Array). All brain tissue samples were from four patients with spontaneous intracerebral hemorrhage who were diagnosed within the previous four days, including two men and two women, with a median age of 79 years (68-92 years), and all samples were obtained within 5 hours of the patients' death. Perihematomal areas were used for the disease groups, while the contralateral grey and white matter were used as controls (Rosell et al. 2011).

\section{Screening results of DEGs}


128 We downloaded the series matrix file dataset of GSE24265, and subsequently transformed the 129 gene probes of the platform to gene names by referencing the GPL570 platform. Both the 130 normalization of the data and screening of the DEGs were performed using the limma package in 131 the R language (version 3.5.1) (Guo et al. 2017). To identify the genes with significant 132 differences in the fold change of expression, we set the screening criteria to filter genes with a 133 fold change $(\mathrm{FC})$ value of $(|\log 2 \mathrm{FC}|)>2$ and a $\mathrm{P}$-value of $<0.05$.

134

GO and KEGG pathway analyses for identification of DEGs

136

137

138

139

140

141

142

143

144

145

146

147

148

149

150

151

152

153

154

155

156

To enable better recognition of the biological functions of DEGs, we used online tools to perform GO enrichment and KEGG analyses. Based on the description of the GO analysis, the gene function annotations were identified as biological processes (BP), cellular components (CC), or molecular functions (MF). DAVID (https://david.ncifcrf.gov/) and KOBAS (version 3.0; http://kobas.cbi.pku.edu.cn/) are considered versatile bioinformatics tools; their main functions include gene annotation, visualization, identification of signaling pathways, and integrated functional discovery. Functional annotation analysis of the DEGs was performed using DAVID, and the KEGG-assisted cell signaling pathway analysis of the DEGs was carried out using KOBAS (Tang et al. 2018). P-values of $<0.05$ were considered to be statistically significant.

\section{Construction of the PPI network and identification of hub genes}

STRING is a public database that contains interactions between known and predicted proteins (https://string-db.org/) (Szklarczyk et al. 2019). PPI is essential for studying protein function since it can help elucidate the function of regulation among proteins. We uploaded the DEGs obtained from the GSE24265 dataset onto STRING's official website to obtain the interrelationships between proteins and to set the minimum required interaction score to 0.15 to visualize the interaction networks with Cytoscape (version 3.2.1) (Shannon et al. 2003). The screening of hub genes was based on the degree of connectivity between DEGs and genes; the DEGs featuring the ten highest degrees were recognized as hub genes. 
157 Obtaining small-molecule compounds for the treatment of ICH by CMap analysis

158 CMap is a gene-expression-based drug development system that explains associations among 159 genes, drugs, and diseases by integrating the effects of thousands of small-molecule drugs on 160 various human cells (Lamb et al. 2006). In this study, we converted the DEGs into probes and 161 subsequently uploaded them onto the official website of CMap to obtain corresponding small162 molecule compounds. The negatively related drugs $(\mathrm{P}<0.01$ and enrichment $<0)$ were 163 considered to be therapeutically effective for treating $\mathrm{ICH}$.

\section{Rat model of ICH}

165 All animals used in this study were tested according to the Guide for Animal Experimentation of 166 Harbin Medical University, and the animal study approved by the Institutional Animal Care and 167 Use Committee at Harbin Medical University. Six adult male Sprague-Dawley rats, weighing 168 200-250 g, aged 8 weeks, provided by the Animal Experimental Center, Harbin Medical 169 University, were used in the present study. The animals, fixed using a stereotaxic frame, were 170 intraperitoneally injected with sodium pentobarbital $(50 \mathrm{mg} / \mathrm{kg})$, and, using a skull drill (about 1 $171 \mathrm{~mm}$ in diameter), holes were drilled near the right coronal suture, $3.5 \mathrm{~mm}$ outside the midline. 172 Autologous whole blood $(50 \mu \mathrm{L})$ was collected from the tail vein using a sterile disposable 173 syringe, and, using the same syringe, the collected autologous blood was slowly injected into 174 the right basal ganglia at a rate of $10 \mu \mathrm{l} / \mathrm{min}$ (He et al. 2018). After ten minutes, the syringe was 175 pulled out and the skin was sutured. Six rats were equally divided into experimental and control 176 groups, but the control group only received a needle insertion. All rats were intraperitoneally 177 injected with a lethal dose of sodium pentobarbital 24 hours after the surgery, and 2-mm thick 178 brain tissue surrounding the path of the injection needle was collected and stored at $-80^{\circ} \mathrm{C}$ until 179 use. All animal experiments conformed to the European Parliament Directive (2010/63/EU) and 180 were approved by the Institutional Animal Care and Use Committee at Harbin Medical 181 University (No. HMUIRB-2008-06).

182

Reverse transcription-quantitative polymerase chain reaction (RT-qPCR) analysis Total RNA from the brain tissue of rats was extracted using Tri ${ }^{\circledR}$-Reagent (Sigma, USA) according to the manufacturer's instructions. The quality and quantity of the RNA was detected using a NanoDrop One spectrophotometer (Thermo Fisher Scientific, USA). Reverse 
187 transcription of the extracted total RNA into cDNA was performed using the Transcriptor First

188 Stand cDNA Synthesis Kit (Roche, USA). RT-qPCR was performed using the FastStart

189 Universal SYBR $®$ Green Master (ROX) (Roche, Germany) and quantified using the

190 QuantStudio software (Thermo Fisher Scientific, USA) according the manufacturer's

191 instructions. GAPDH was used as the endogenous reference gene. The sequence of all primers

192 used in this study is provided in supplementary Table 1. The expression level of all mRNAs was

193 determined using the $2^{-\Delta \mathrm{CT}}$ method. A meaningful analysis between the two groups was

194 performed by a paired t-test, and a P-value $<0.05$ was considered statistically significant.

195

196

197

198

199

200

201

202

203

204

205

206

207

208

209

210

211

212 213 meaningful functional annotation analyses are summarized in Table 3 and Figure 2.

\section{Results}

\section{Identification of DEGs in ICH}

The gene expression profile of the GSE24265 dataset contained data obtained from four perihematomal tissues and seven contralateral tissues (Table 1). The result of the normalization of data among the arrays of the series matrix files of the GSE24265 dataset is shown in Supplementary Figure 1. Using a P-value of $<0.05$ and a $[\operatorname{logFC}]$ of $>2$ as the cut-off, a total of 67 DEGs, including 59 up-regulated and 8 down-regulated genes, were obtained on comparison of the expression profiles between the cerebral hemorrhage and control groups (Table 2). Volcano plots and heat maps for the distribution of DEGs were generated using R software (Figures $1 \mathrm{~A}$ and $\mathrm{B})$.

\section{Functional annotation analysis of DEGs using GO}

On GO analysis, the three most significant processes identified among the annotations of BP were inflammatory response, neutrophil chemotaxis, and immune response. In parallel, the three most significant processes identified among the annotations of MF were chemokine activity,

10 CXCR chemokine receptor binding, and growth factor activity. Finally, the three most 11 significant processes identified among the annotations of CC were extracellular space, 12 extracellular region, and plasma membrane. According to the criteria, the results of all

\section{KEGG pathway analysis of DEGs}


215 The results of the cell signaling pathway enrichment analysis of the DEGs identified a total of 29

216 meaningful pathways which were analyzed. The cellular signaling pathways clearly associated

217 with brain damage included those for HIF-1, TNF, toll-like receptor, the NF-kappa B signaling

218 pathway, cytokine-cytokine receptor interaction, and chemokine signaling pathways. The

219 specific enriched pathways obtained from the analysis of the DEGs are summarized in Table 4 220 and Figure 3.

221

222

223

224

225

226

227

228

229

230

231

232

233

234

235

236

237

238

239

240

241

242

\section{PPI network construction and hub gene identification}

We uploaded 67 DEGs to the string online database to obtain the PPI network. A total of 57 DEGs were consequently extracted among the 67 DEGs uploaded when the interaction score was set to 0.15 . The resulting PPI network contained a total of 57 nodes and 373 edges. Figure 4A presents the visualization of the network generated using Cytoscape software; Figure 4B represents the ten selected hub genes with the highest degrees of connectivity that were selected as the hub genes. Prior reports indicate that the identified hub genes are closely related to ICH and brain damage. The subsequent GO function annotation analysis and KEGG pathway analysis results for hub genes are summarized in Table 5 and Table 6.

\section{CMap analysis}

We used CMap to analyze the previously selected DEGs and to identify small-molecule compounds with potential therapeutic application in ICH cases. Nine small-molecule compounds which exhibited high correlation with ICH are shown in Table 7. The 3-dimensional chemical structures of the small-molecule compounds hecogenin, lidocaine, and NU-1025, previously reported to be significantly associated with brain damage, are shown in Figure 5.

\section{Corroboration of ten hub genes using RT-qPCR}

RT-qPCR was performed with the total RNA extracted from the brain tissue of three pairs of rats used for the ICH model to confirm the expression levels of ten hub genes. According to the PPI network analysis, the ten core genes screened were IL6, TLR2, CXCL1, TIMP1, PLAUR, SERPINE1, SELE, CCL4, CCL20, and CD163. The expression levels of the ten hub genes were increased as part of the pathophysiological process of ICH. The results of the RT-qPCR experiment showed a significant increase in the expression of the hub genes (Figure 6). 


\section{Discussion}

245 The present study uses the GEO database to obtain gene expression profiles from patients with

246 ICH and screens for DEGs. We perform functional enrichment analyses on the obtained DEGs to

247 understand their biological functions. Meanwhile, we report meaningful enrichment, by KEGG

248 analysis, of pathways involved in triggering the regulatory pathway associated with secondary

249 brain injury induced by ICH. Next, PPI analyses were carried out to identify the hub genes that

250 may play a crucial regulatory role in the pathophysiology of ICH. At the same time, we

251 established a rat model of $\mathrm{ICH}$, and confirmed that the expression level of hub genes were

252 consistent with the results of our analyses by RT-qPCR. In addition, several potential small-

253 molecule compounds, related to ICH, were identified by CMap analysis. These may become

254 effective drugs for treating ICH in future.

255

256

257

258

259

260

261

262

263

264

265

266

267

268

269

270

271

272

273

From the GSE24265 dataset, after comparing the gene expression data obtained from ICH tissue with that obtained from the contralateral brain tissues, we obtained 67 DEGs. GO analysis indicated that the main functional annotation results were consistent with those obtained by previous reports, i.e., a significant increase in inflammatory response promotes ICH-induced brain injury, which may be one of the main factors contributing to the poor prognosis in patients with ICH (Boehme et al. 2018; Fu et al. 2018). The immune response can antagonize the inflammatory response, induced after an episode of cerebral hemorrhage, to reduce the secondary damage to brain tissue, and thereby improve the recovery of nerve function (Klebe et al. 2015; $\mathrm{Lu}$ et al. 2014). Previous reports have indicated that the cellular signaling pathways identified in this study using a KEGG analysis were closely related to cerebral hemorrhage; those activated within a few hours after cerebral hemorrhage, including the TNF signaling pathway, which participates in the secondary inflammatory reactions and formation of cerebral edema around the hematoma (Testai \& Aiyagari 2008) and the HIF-1 signaling pathway, which contributes to the recovery of neural function after ICH by regulating the proliferation and differentiation of neural stem cells (Yu et al. 2013). The NF-kappa B signaling pathway, which is closely related to the death of cells surrounding the hematoma in the animal ICH model, and the activation of which is associated with deficiencies in neurological function (Zhou et al. 2014), was activated several minutes after the ICH event. The Toll-like receptor signaling pathway is significantly associated with a poor prognosis after ICH because it promotes the inflammatory response of ICH-induced 
274 brain injury (Wang et al. 2014b). Therefore, prior reports have been able to verify the accuracy 275 of our results.

276 PPI networks are essential in most biological processes, play an important role in the 277 development of many diseases and are very useful for the identification of drug targets, therefore 278 demonstrating great potential for drug discovery (Rabbani et al. 2018). Compared to the original 279 article which identified ICH related genes only by multiple gene expression analyses (Rosell et 280 al. 2011), hub genes screened by constructing PPI networks are more relevant to the pathological 281 processes of ICH and are more likely to be potential therapeutic targets. Therefore, we uploaded 282 the DEGs to the STRING database to build a PPI network and further identify the hub genes that 283 influence regulatory processes in the pathophysiology of ICH-induced brain injury. For instance, 284 IL-6 was significantly elevated in the serum of patients with ICH, and its levels were clearly 285 associated with Glasgow Coma Scale scores (Dziedzic et al. 2002). During brain injury, IL-6 286 signaling may not only induce the chemotactic migration of inflammatory cells to the site of 287 lesion but may also promote T-cell polarization (Chaudhry et al. 2017). The inflammatory 288 response plays a key role in ICH-induced brain injury, and hemoglobin causes toll-like receptors 2892 and 4 to heterodimerize and aggravate brain damage (Wang et al. 2014b). Within 24 hours of 290 having a stroke, the expression level of CXCL1 in the cerebrospinal fluid of patients positively 291 correlates with the ischemic area, indicating that CXCL1 may participate in the regulation of 292 brain tissue damage (Losy et al. 2005). The abnormal expression of TIMP1 can disrupt the 293 integrity of the blood-brain barrier and this disruption may be involved in the regulation of the 294 pathological process of ICH (Wang et al. 2014a). The urokinase-type plasminogen activator, 295 encoded by the PLAUR gene, is involved in the development of the neural circuit of the cortex and in brain tissue remodeling after a brain injury (Levi et al. 2001). A few hours after the onset of ICH, because of red blood cell fragmentation, some products released by the lysed red blood cells around the site of the hematoma were involved in secondary brain damage. In this process, 299 CD163 and hemoglobin form a complex which participates in the pathological process of 300 secondary brain injury induced by ICH (Lieber \& Mocco 2018). SERPINE1, CCL4, and CCL20 301 have not yet been reported in cases of IHC, but, on review of the literature, we found that these 302 303 genes are associated with nerve injury, such as sterile inflammatory reaction and traumatic brain injury, which are pathologically similar to ICH, such as sterility inflammatory reaction (Graber 
304 et al. 2015; Kwiecien 2018; Leonardo et al. 2012). These hub genes, therefore, feature as

305 potential prognostic or diagnostic markers, as well as potential therapeutic targets.

306 CMap is a database that covers the interactions among diseases, gene expression patterns, and

307 small-molecule compounds. It is often used to screen for small-molecule compounds with

308 therapeutic effects on some diseases (Lamb et al. 2006). Using CMap analysis, we identified

309 nine small molecule drugs, two of which were lidocaine and NU-1025, both previously reported

310 to have a salutary effect in cases of brain injury. Lidocaine is commonly used as a local

311 anesthetic and has been shown to have significant anti-inflammatory effects. A recent study

312 demonstrated that lidocaine inhibited the release of inflammatory factors and alleviated

313 microglial damage (Jeong et al. 2012). Moreover, lidocaine is considered to have a therapeutic

314 effect in cases of nerve damage induced by cerebral hemorrhage. Recent studies have found that

315 NU-1025, a poly (ADP-ribose) polymerase inhibitors, can attenuate neurological damage in

316 animal models of ischemic stroke and traumatic brain injury; moreover, rats treated with

317 NU1025 exhibited reduced infarction, significantly reduced edema volume, and significantly

318 ameliorated neurological impairment (Kaundal et al. 2006). Thus, NU-1025 can both mitigate

319 the damage inflicted in the early stages of nerve injury and reduce the degree of subsequent

320 neuroinflammation (Curtin \& Szabo 2013). Although the two aforementioned drugs have been

321 reported to be associated with the treatment of $\mathrm{ICH}$, the mechanisms underlying their therapeutic

322 actions still require further research. In addition, we found that hecogenin has an anti-

323 inflammatory effect that may contribute to the treatment of ICH (Ingawale et al. 2019; Ingawale

324 \& Patel 2016; Ingawale \& Patel 2018).

325 However, this study had several limitations. First, the mechanisms of several hub genes in the 326 pathological process of ICH remain unclear, warranting further study. Moreover, the

327 effectiveness of our small molecule compound screening in reducing ICH-induced brain injury

328 remains to be assessed.

\section{Conclusions}

330 The hub genes identified by constructing a PPI network have potential in the development of 331 new targets in the treatment of ICH and as prognostic markers in patients with ICH. The hub 332 genes may be involved in the pathological process of ICH and ultimately affect their prognosis 333 through TNF signaling pathway, HIF-1 signaling pathway, NF-kappa B signaling pathway, Toll- 
334 like receptor signaling pathway and Chemokine signaling pathway. In addition, this study

335 identified small-molecule compounds that may be efficacious in the treatment of secondary brain 336 injury induced by ICH.

\section{Acknowledgements}

338 This work was supported China Postdoctoral Science Foundation (2017M620119); Heilongjiang

339 Postdoctoral Fund (LBH-Z17108) and the Scientific Research Project of Heilongjiang Provincial

Department of Health (Nos.2013030).

\section{References}

Akira S, and Takeda K. 2004. Toll-like receptor signalling. Nat Rev Immunol 4:499-511. $10.1038 /$ nri1391

Aronowski J, and Hall CE. 2005. New horizons for primary intracerebral hemorrhage treatment: experience from preclinical studies. Neurol Res 27:268-279. 10.1179/016164105X25225

Aronowski J, and Zhao X. 2011. Molecular pathophysiology of cerebral hemorrhage: secondary brain injury. Stroke 42:1781-1786. 10.1161/STROKEAHA.110.596718

Boehme AK, Comeau ME, Langefeld CD, Lord A, Moomaw CJ, Osborne J, James ML, Martini S, Testai FD, Woo D, and Elkind MSV. 2018. Systemic inflammatory response syndrome, infection, and outcome in intracerebral hemorrhage. Neurol Neuroimmunol Neuroinflamm 5:e428. 10.1212/NXI.0000000000000428

Chaudhry SR, Stoffel-Wagner B, Kinfe TM, Guresir E, Vatter H, Dietrich D, Lamprecht A, and Muhammad S. 2017. Elevated Systemic IL-6 Levels in Patients with Aneurysmal Subarachnoid Hemorrhage Is an Unspecific Marker for Post-SAH Complications. Int J Mol Sci 18. 10.3390/ijms18122580

Clough E, and Barrett T. 2016. The Gene Expression Omnibus Database. Methods Mol Biol 1418:93-110. 10.1007/978-1-4939-3578-9 5

Curtin NJ, and Szabo C. 2013. Therapeutic applications of PARP inhibitors: anticancer therapy and beyond. Mol Aspects Med 34:1217-1256. 10.1016/j.mam.2013.01.006

Dziedzic T, Bartus S, Klimkowicz A, Motyl M, Slowik A, and Szczudlik A. 2002. Intracerebral hemorrhage triggers interleukin-6 and interleukin-10 release in blood. Stroke 33:23342335.

Fogelholm R, Murros K, Rissanen A, and Avikainen S. 2005. Long term survival after primary intracerebral haemorrhage: a retrospective population based study. J Neurol Neurosurg Psychiatry 76:1534-1538. 10.1136/jnnp.2004.055145

Fu G, Wang H, Cai Y, Zhao H, and Fu W. 2018. Theaflavin alleviates inflammatory response and brain injury induced by cerebral hemorrhage via inhibiting the nuclear transcription factor kappa beta-related pathway in rats. Drug Des Devel Ther 12:1609-1619. 10.2147/DDDT.S164324

Graber DJ, Costine BA, and Hickey WF. 2015. Early inflammatory mediator gene expression in two models of traumatic brain injury: ex vivo cortical slice in mice and in vivo cortical impact in piglets. J Neuroinflammation 12:76. 10.1186/s12974-015-0298-4

Guo W, Zhang B, Li Y, Duan HQ, Sun C, Xu YQ, and Feng SQ. 2017. Gene expression profile identifies potential biomarkers for human intervertebral disc degeneration. Mol Med Rep 16:8665-8672. 10.3892/mmr.2017.7741 
377

378

379

380

381

382

383

384

385

386

387

388

389

390

391

392

393

394

395

396

397

398

399

400

401

402

403

404

405

406

407

408

409

410

411

412

413

414

415

416

417

418

419

420

421

422

423

424

425

426

427

He M, Wang Y, Shen J, Duan C, Lu X, and Li J. 2018. Bex1 attenuates neuronal apoptosis in rat intracerebral hemorrhage model. Pathol Res Pract 214:527-535. 10.1016/j.prp.2018.02.012

Hua Y, Wu J, Keep RF, Nakamura T, Hoff JT, and Xi G. 2006. Tumor necrosis factor-alpha increases in the brain after intracerebral hemorrhage and thrombin stimulation. Neurosurgery 58:542-550; discussion 542-550. 10.1227/01.NEU.0000197333.55473.AD

Ingawale DK, Mandlik SK, and Patel SS. 2019. Anti-inflammatory potential of hecogenin on atopic dermatitis and airway hyper-responsiveness by regulation of pro-inflammatory cytokines. Immunopharmacol Immunotoxicol 41:327-336. 10.1080/08923973.2019.1608445

Ingawale DK, and Patel SS. 2016. Anti-In fl ammatory Potential of Hecogenin in Experimental Animals: Possible Involvement of Inflammatory Cytokines and Myeloperoxidase. Drug Res (Stuttg) 66:644-656. 10.1055/s-0042-113184

Ingawale DK, and Patel SS. 2018. Hecogenin exhibits anti-arthritic activity in rats through suppression of pro-inflammatory cytokines in Complete Freund's adjuvant-induced arthritis. Immunopharmacol Immunotoxicol 40:59-71. 10.1080/08923973.2017.1405439

Jeong HJ, Lin D, Li L, and Zuo Z. 2012. Delayed treatment with lidocaine reduces mouse microglial cell injury and cytokine production after stimulation with lipopolysaccharide and interferon gamma. Anesth Analg 114:856-861. 10.1213/ANE.0b013e3182460ab5

Kaundal RK, Shah KK, and Sharma SS. 2006. Neuroprotective effects of NU1025, a PARP inhibitor in cerebral ischemia are mediated through reduction in NAD depletion and DNA fragmentation. Life Sci 79:2293-2302. 10.1016/j.lfs.2006.07.034

Klebe D, McBride D, Flores JJ, Zhang JH, and Tang J. 2015. Modulating the Immune Response Towards a Neuroregenerative Peri-injury Milieu After Cerebral Hemorrhage. $J$ Neuroimmune Pharmacol 10:576-586. 10.1007/s11481-015-9613-1

Kwiecien JM. 2018. Methods for Assessing Serpins as Neuroprotective Therapeutics. Methods Mol Biol 1826:223-235. 10.1007/978-1-4939-8645-3_15

Labovitz DL, Halim A, Boden-Albala B, Hauser WA, and Sacco RL. 2005. The incidence of deep and lobar intracerebral hemorrhage in whites, blacks, and Hispanics. Neurology 65:518522. 10.1212/01.wnl.0000172915.71933.00

Lamb J, Crawford ED, Peck D, Modell JW, Blat IC, Wrobel MJ, Lerner J, Brunet JP, Subramanian A, Ross KN, Reich M, Hieronymus H, Wei G, Armstrong SA, Haggarty SJ, Clemons PA, Wei R, Carr SA, Lander ES, and Golub TR. 2006. The Connectivity Map: using gene-expression signatures to connect small molecules, genes, and disease. Science 313:1929-1935. 10.1126/science.1132939

Leonardo CC, Musso J, Das M, Rowe DD, Collier LA, Mohapatra S, and Pennypacker KR. 2012. CCL20 Is Associated with Neurodegeneration Following Experimental Traumatic Brain Injury and Promotes Cellular Toxicity In Vitro. Trans/ Stroke Res 3:357-363. 10.1007/s12975-012-0203-8

Levi M, Moons L, Bouche A, Shapiro SD, Collen D, and Carmeliet P. 2001. Deficiency of urokinase-type plasminogen activator-mediated plasmin generation impairs vascular remodeling during hypoxia-induced pulmonary hypertension in mice. Circulation 103:2014-2020.

Lieber AC, and Mocco J. 2018. CD163, Hemoglobin, and Secondary Brain Injury After Intracerebral Hemorrhage. World Neurosurg 117:459. 10.1016/j.wneu.2018.07.111

Losy J, Zaremba J, and Skrobanski P. 2005. CXCL1 (GRO-alpha) chemokine in acute ischaemic stroke patients. Folia Neuropathol 43:97-102.

Lu Q, Gao L, Huang L, Ruan L, Yang J, Huang W, Li Z, Zhang Y, Jin K, and Zhuge Q. 2014. Inhibition of mammalian target of rapamycin improves neurobehavioral deficit and modulates immune response after intracerebral hemorrhage in rat. $J$ Neuroinflammation 11:44. 10.1186/1742-2094-11-44

Peer] reviewing PDF | (2019:04:36749:2:0:NEW 16 Aug 2019) 
428

429

430

431

432

433

434

435

436

437

438

439

440

441

442

443

444

445

446

447

448

449

450

451

452

453

454

455

456

457

458

459

460

461

462

463

464

465

466

467

468

469

470

471

472

473

474

475

476

477

478

Morioka M, and Orito K. 2017. Management of Spontaneous Intracerebral Hematoma. Neurol Med Chir (Tokyo) 57:563-574. 10.2176/nmc.ra.2016-0327

Qureshi Al, Suri MF, Nasar A, Kirmani JF, Ezzeddine MA, Divani AA, and Giles WH. 2007. Changes in cost and outcome among US patients with stroke hospitalized in 1990 to 1991 and those hospitalized in 2000 to 2001. Stroke 38:2180-2184. 10.1161/STROKEAHA.106.467506

Qureshi Al, Tuhrim S, Broderick JP, Batjer HH, Hondo H, and Hanley DF. 2001. Spontaneous intracerebral hemorrhage. N Engl J Med 344:1450-1460. 10.1056/NEJM200105103441907

Rabbani G, Baig MH, Ahmad K, and Choi I. 2018. Protein-protein Interactions and their Role in Various Diseases and their Prediction Techniques. Curr Protein Pept Sci 19:948-957. 10.2174/1389203718666170828122927

Rodriguez-Yanez M, Brea D, Arias S, Blanco M, Pumar JM, Castillo J, and Sobrino T. 2012. Increased expression of Toll-like receptors 2 and 4 is associated with poor outcome in intracerebral hemorrhage. J Neuroimmunol 247:75-80. 10.1016/j.jneuroim.2012.03.019

Rosell A, Vilalta A, Garcia-Berrocoso T, Fernandez-Cadenas I, Domingues-Montanari S, Cuadrado E, Delgado P, Ribo M, Martinez-Saez E, Ortega-Aznar A, and Montaner J. 2011. Brain perihematoma genomic profile following spontaneous human intracerebral hemorrhage. PLoS One 6:e16750. 10.1371/journal.pone.0016750

Shannon P, Markiel A, Ozier O, Baliga NS, Wang JT, Ramage D, Amin N, Schwikowski B, and Ideker T. 2003. Cytoscape: a software environment for integrated models of biomolecular interaction networks. Genome Res 13:2498-2504. 10.1101/gr.1239303

Szklarczyk D, Gable AL, Lyon D, Junge A, Wyder S, Huerta-Cepas J, Simonovic M, Doncheva NT, Morris JH, Bork P, Jensen LJ, and Mering CV. 2019. STRING v11: protein-protein association networks with increased coverage, supporting functional discovery in genome-wide experimental datasets. Nucleic Acids Res 47:D607-D613. 10.1093/nar/gky1131

Takeda K, and Akira S. 2004. TLR signaling pathways. Semin Immunol 16:3-9.

Tang X, Xu Y, Lu L, Jiao Y, Liu J, Wang L, and Zhao H. 2018. Identification of key candidate genes and small molecule drugs in cervical cancer by bioinformatics strategy. Cancer Manag Res 10:3533-3549. 10.2147/CMAR.S171661

Testai FD, and Aiyagari V. 2008. Acute hemorrhagic stroke pathophysiology and medical interventions: blood pressure control, management of anticoagulant-associated brain hemorrhage and general management principles. Neurol Clin 26:963-985, viii-ix. 10.1016/j.ncl.2008.06.001

Wagner KR. 2007. Modeling intracerebral hemorrhage: glutamate, nuclear factor-kappa B signaling and cytokines. Stroke 38:753-758. 10.1161/01.STR.0000255033.02904.db

Wang HX, Yang QD, Liu BQ, Zhang L, Ma MM, Hu ZY, Xia J, Xu HW, and Du XP. 2014a. TIMP-1 polymorphisms in a Chinese Han population with intracerebral hemorrhage. Int J Neurosci 124:61-67. 10.3109/00207454.2013.823604

Wang J, and Dore S. 2007. Inflammation after intracerebral hemorrhage. J Cereb Blood Flow Metab 27:894-908. 10.1038/sj.jcbfm.9600403

Wang YC, Zhou Y, Fang H, Lin S, Wang PF, Xiong RP, Chen J, Xiong XY, Lv FL, Liang QL, and Yang QW. 2014b. Toll-like receptor 2/4 heterodimer mediates inflammatory injury in intracerebral hemorrhage. Ann Neurol 75:876-889. 10.1002/ana.24159

Yin Y, Ge H, Zhang JH, and Feng H. 2017. Targeting Vascular Neural Network in Intracerebral Hemorrhage. Curr Pharm Des 23:2197-2205. 10.2174/1381612822666161027122821

Yu Z, Chen LF, Tang L, and Hu CL. 2013. Effects of recombinant adenovirus-mediated hypoxiainducible factor-1alpha gene on proliferation and differentiation of endogenous neural stem cells in rats following intracerebral hemorrhage. Asian Pac J Trop Med 6:762-767. 10.1016/S1995-7645(13)60134-0

Peer] reviewing PDF | (2019:04:36749:2:0:NEW 16 Aug 2019) 
479 Zhao X, Grotta J, Gonzales N, and Aronowski J. 2009. Hematoma resolution as a therapeutic 480 481 target: the role of microglia/macrophages. Stroke 40:S92-94. 10.1161/STROKEAHA. 108.533158

Zhao X, Sun G, Zhang J, Strong R, Song W, Gonzales N, Grotta JC, and Aronowski J. 2007. Hematoma resolution as a target for intracerebral hemorrhage treatment: role for peroxisome proliferator-activated receptor gamma in microglia/macrophages. Ann Neurol 61:352-362. 10.1002/ana.21097

Zhou Y, Wang Y, Wang J, Anne Stetler R, and Yang QW. 2014. Inflammation in intracerebral hemorrhage: from mechanisms to clinical translation. Prog Neurobiol 115:25-44.

489 10.1016/j.pneurobio.2013.11.003 


\section{Figure 1}

\section{Comparison of DEGs present in ICH and normal samples.}

(A) The volcano plot for DEGs in GSE24265 data. X-axes index the -log (P-value) and y-axes index the log fold change. The red dots represent upregulated genes and the green dots represent downregulated genes. The black dots represent genes with no significant difference. FC is the fold change.

(B) The expression data is represented as a data matrix wherein each row represents a gene and each column represents a sample. The blue coded bar above the heat map represents the normal sample set and the red coded bar represents the perihematomal sample. The expression level is described in terms of the color ratio of the upper left corner. Hierarchical clustering is shown by the top tree view, indicating the degree of relatedness in gene expression.

Abbreviations: DEG, differentially expressed genes; ICH, intracerebral hemorrhage; FC, fold change.
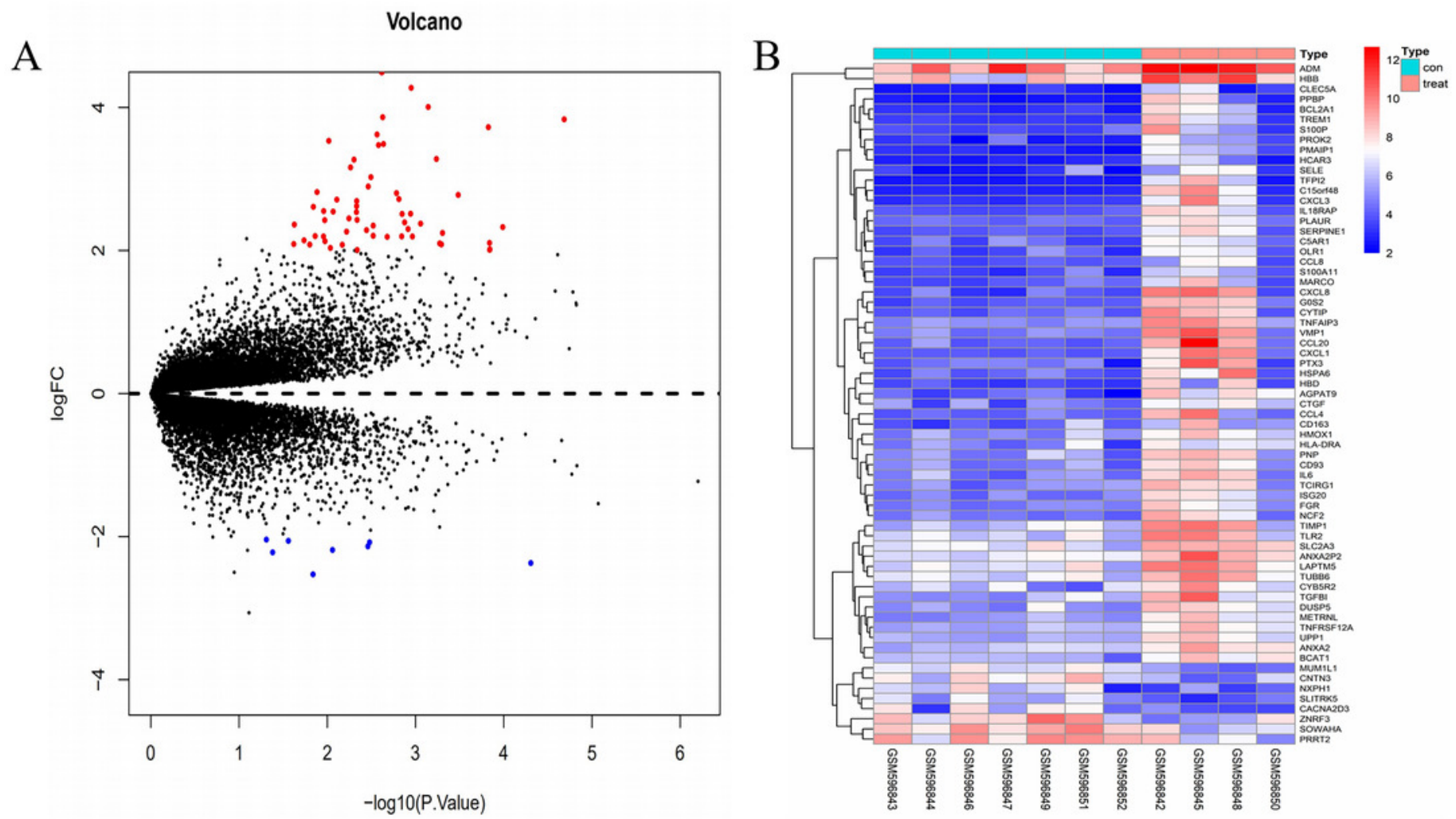
Figure 2

Results of GO enrichment.

The abscissa represents the enriched GO, and the ordinate represents the number and ratio of the differentially expressed genes. Different colors represent different GO classes:

Molecular function, Biological process, and Cellular component. Abbreviation: GO, gene ontology. 


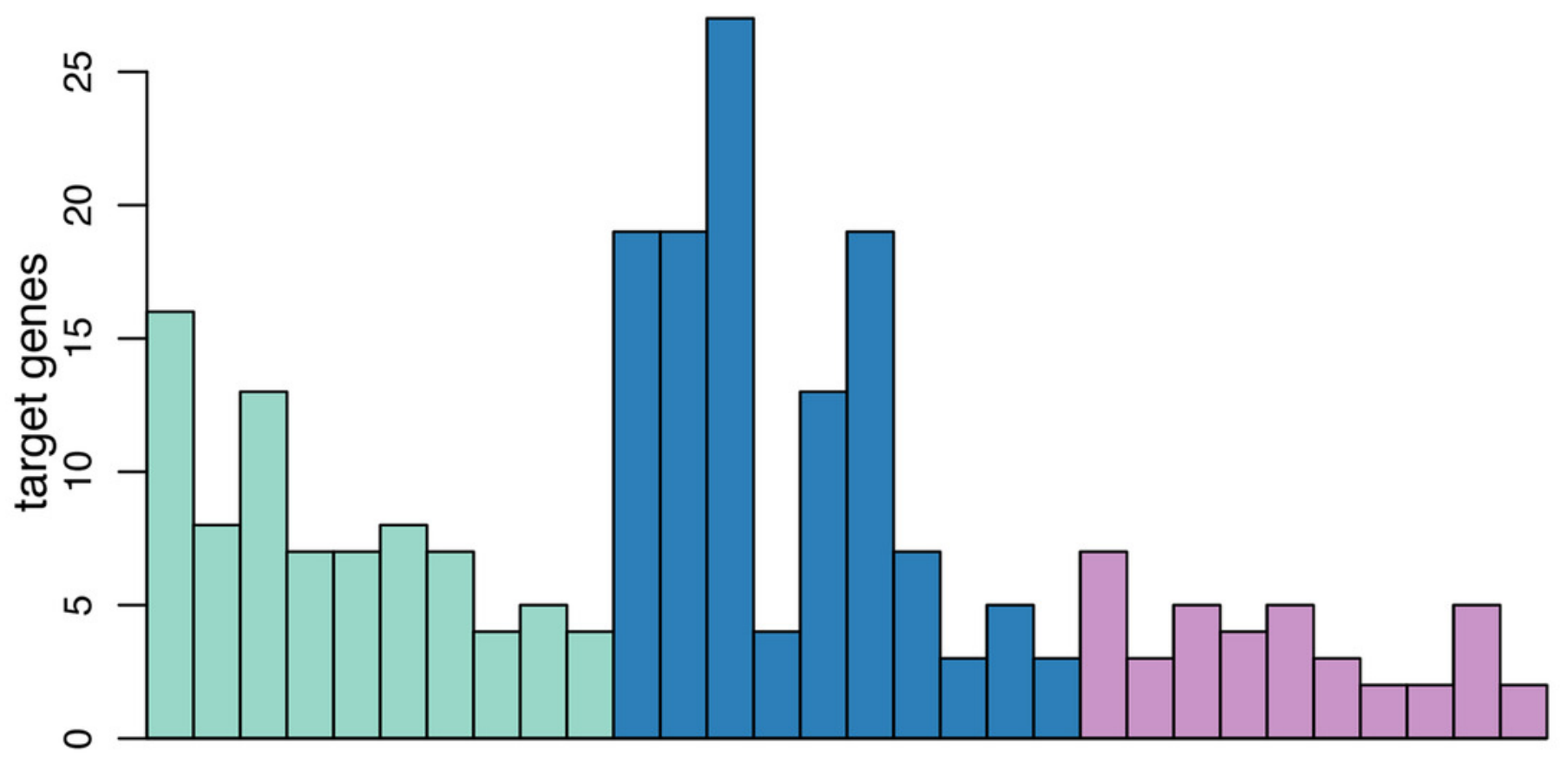

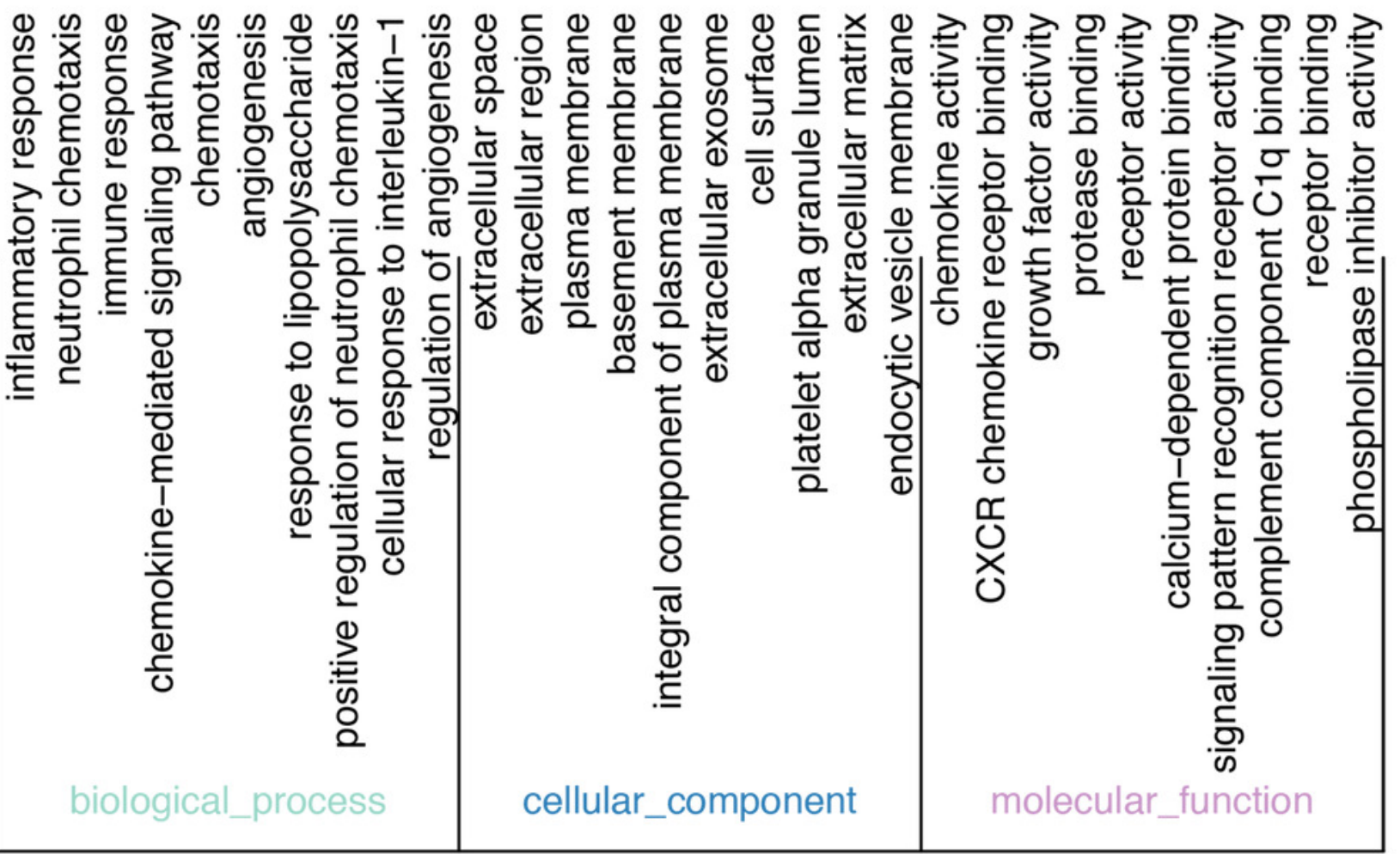


Figure 3

KEGG pathway analysis of the differentially expressed genes in ICH.

Abbreviations: KEGG, Kyoto Encyclopedia of Genes and Genomes; ICH, intracerebral hemorrhage.

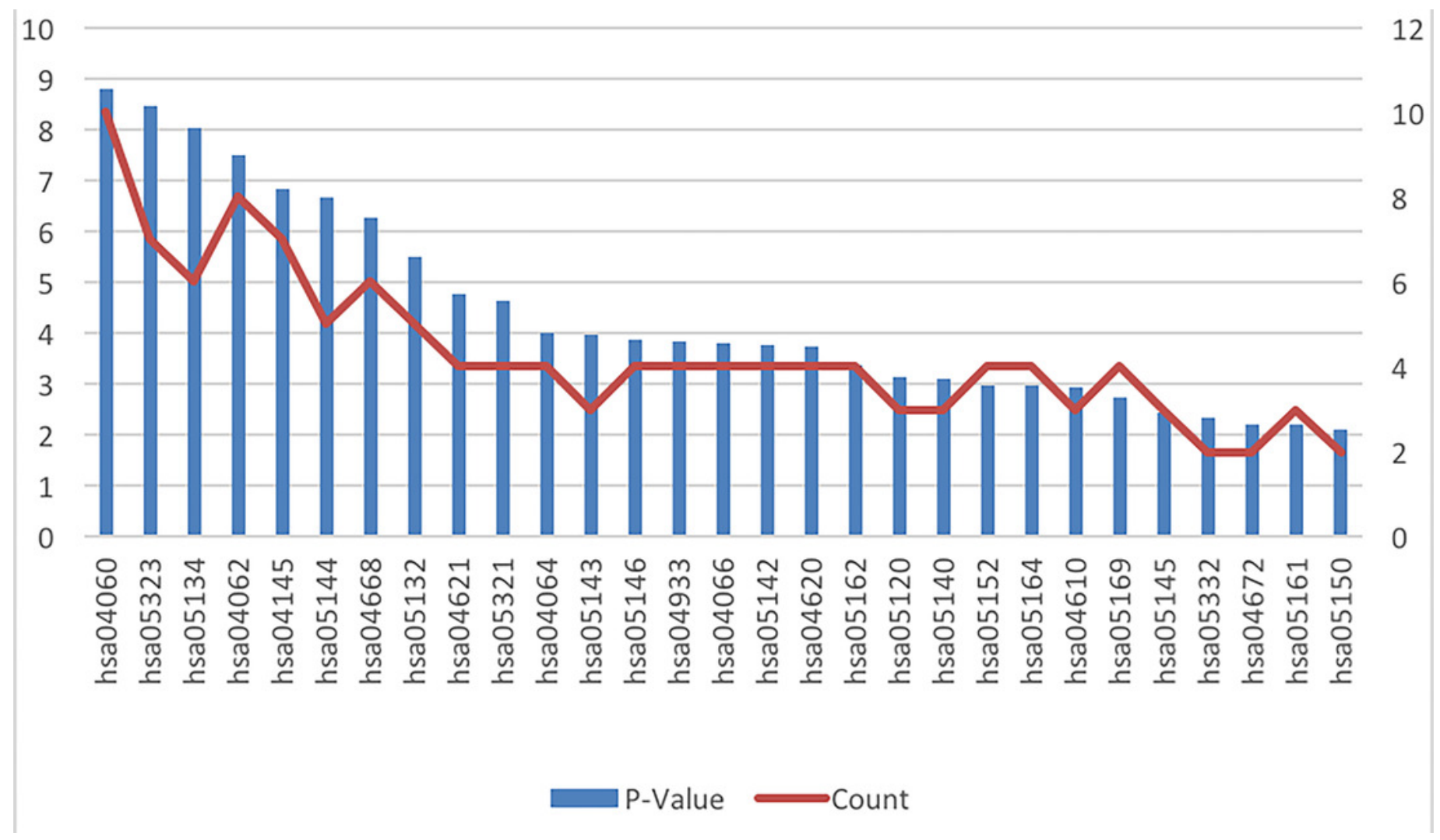


Figure 4

PPI network analysis.

(A). Using the STRING online database, a total of 57 DEGs (56 upregulated [red] and one downregulated gene [green]) were filtered into the DEG PPI network. (B) Of the DEGs with more than ten edges in the PPI network, the ten with the highest degrees of connectivity were selected as the hub genes; all are upregulated genes.

Abbreviations: PPI, protein-protein interaction; DEGs, differentially expressed genes.

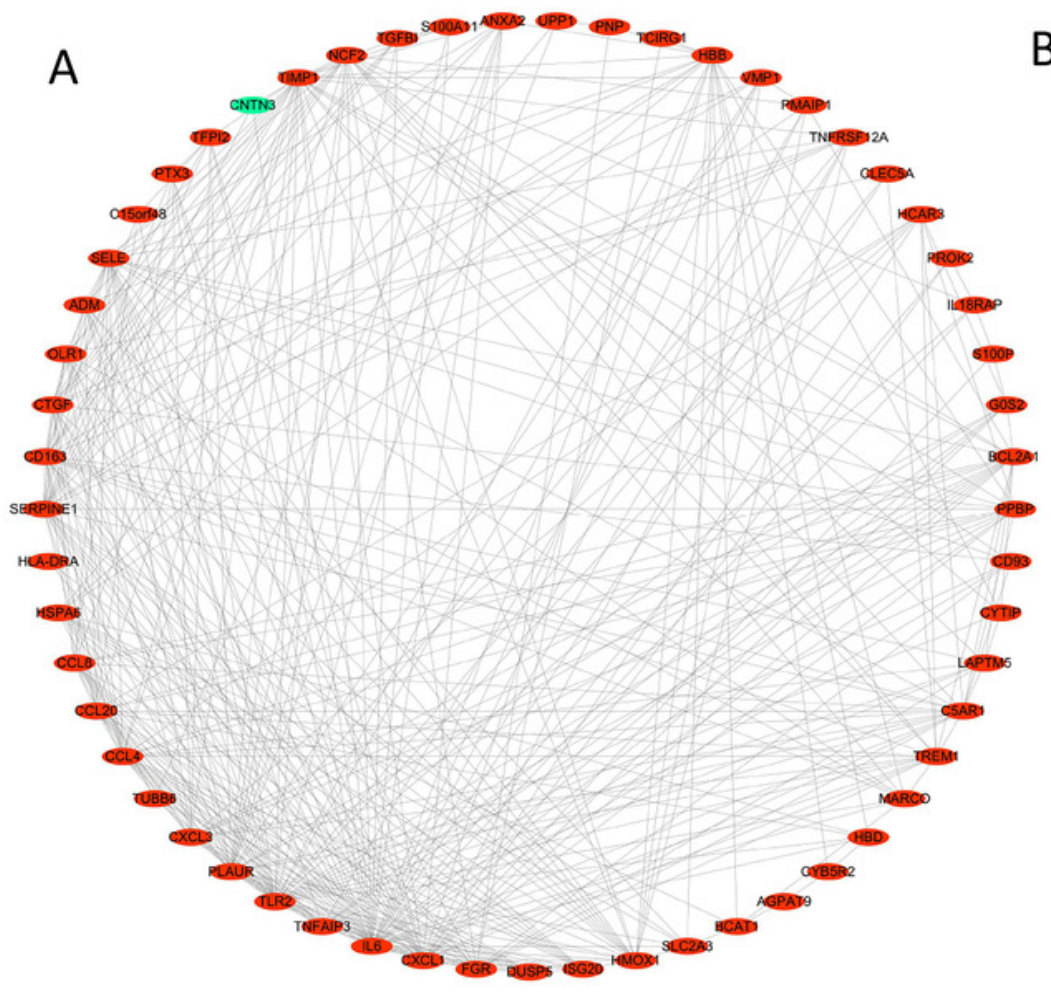

B

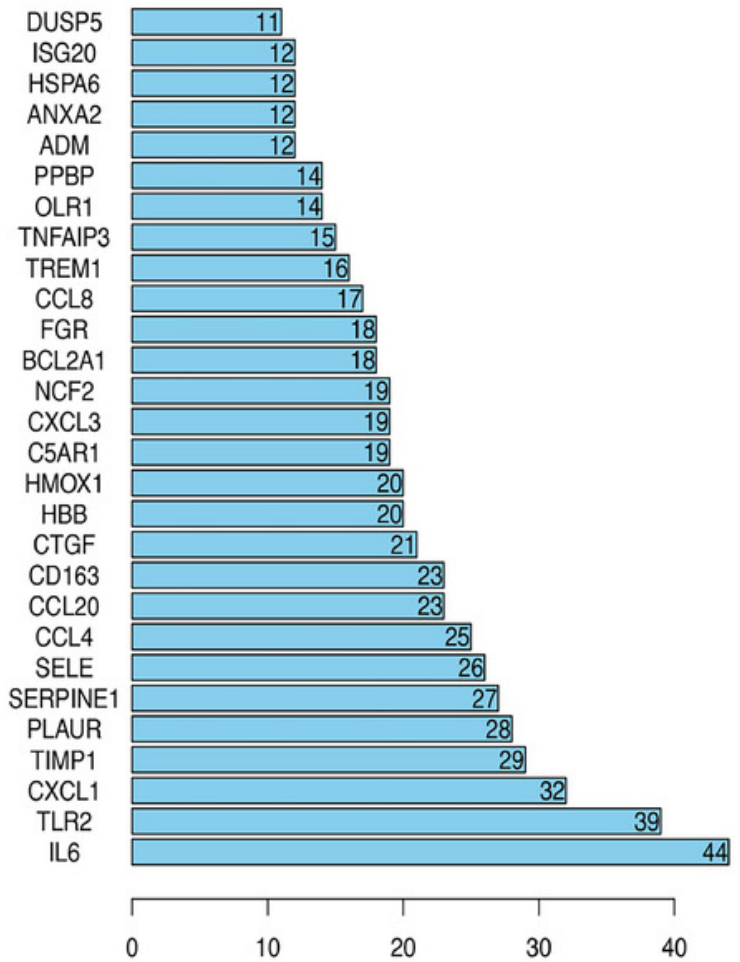


Figure 5

The 3D conformers of the three compounds that may attenuate secondary injury after hematoma.

The 3D structures of the nine compounds were provided by PubChem (https: // pubchem.ncbi.nlm.nih.gov/compound).

A

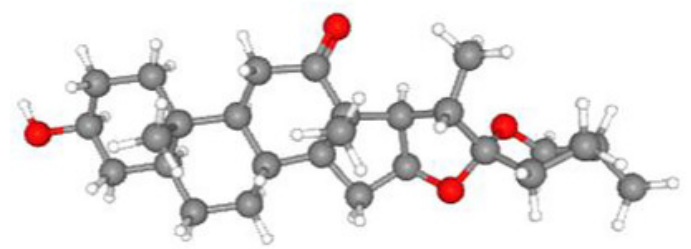

B

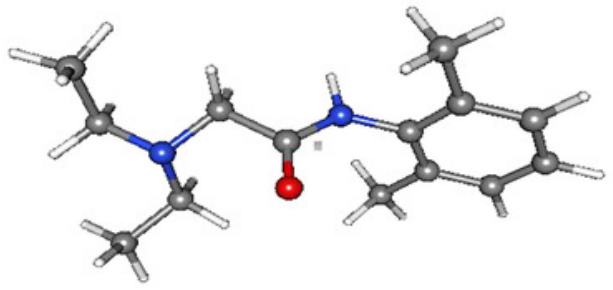

$\mathrm{C}$

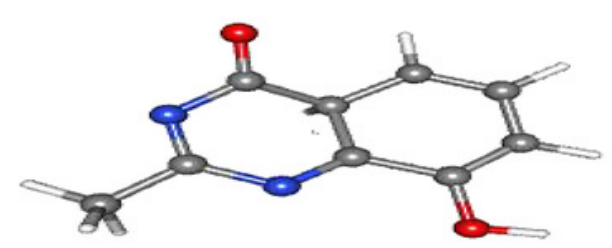

Hecogenin

Molecular Formula: $\mathrm{C}_{27} \mathrm{H}_{42} \mathrm{O}_{4}$

PubChem CID:91453

Lidocaine

Molecular Formula: $\mathrm{C}_{14} \mathrm{H}_{22} \mathrm{~N}_{2} \mathrm{O}$

PubChem CID:3676

NU-1025

Molecular Formula: $\mathrm{C}_{9} \mathrm{H}_{8} \mathrm{~N}_{2} \mathrm{O}_{2}$ PubChem CID:135398517 
Figure 6

Relative expression of the ten hub genes including IL6, TLR2, CXCL1, TIMP1, PLAUR, SERPINE1, SELE, CCL4, CCL20, and CD163 measured by RT-qPCR. 

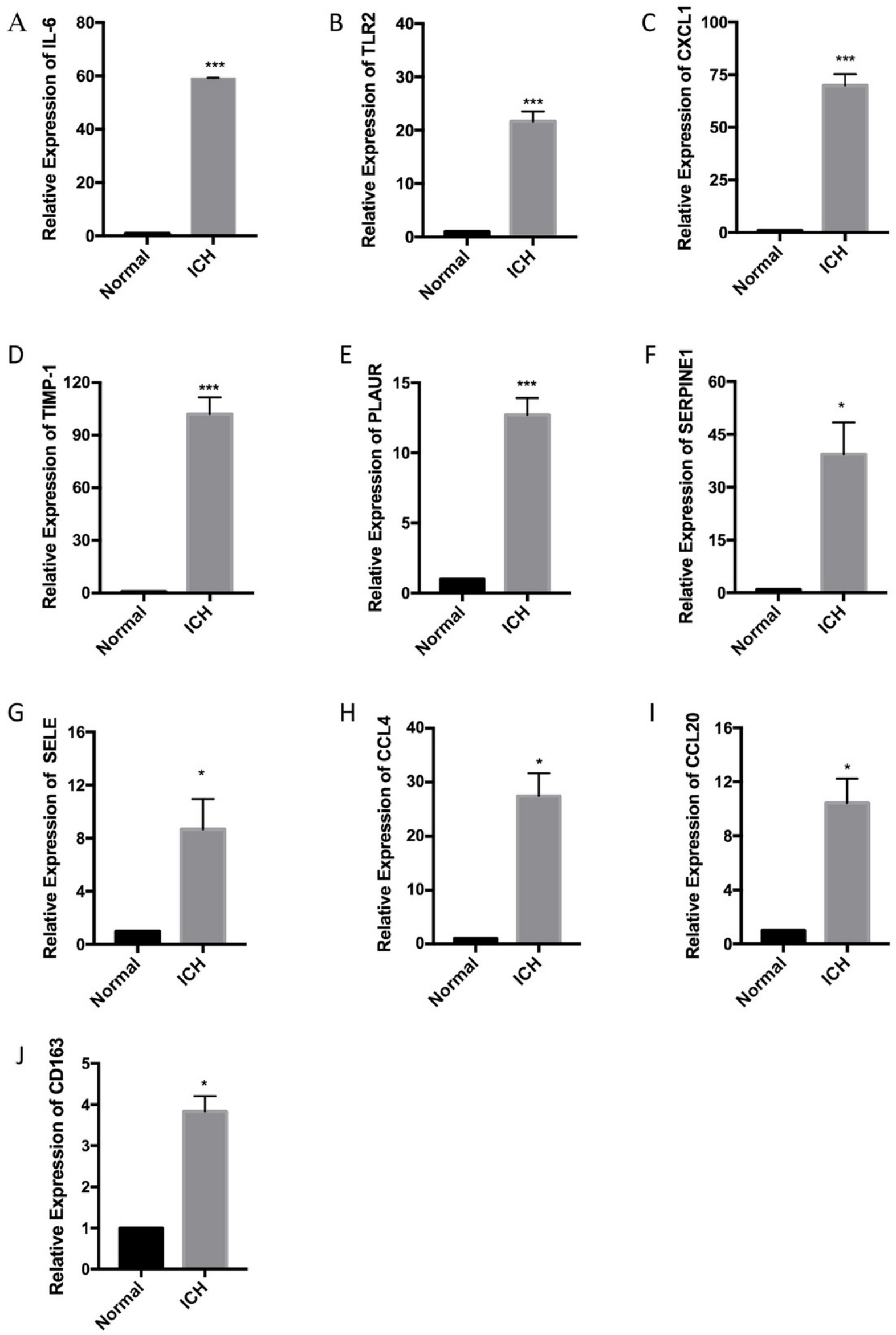
Table $\mathbf{1}$ (on next page)

Details for GEO ICH data.

PH: perihematomal area. CW: contralateral white matter. CG: contralateral grey matter 
Table 1 Details for GEO ICH data.

\begin{tabular}{lllllll}
\hline Reference & Sample & GEO & Platform & PH & CW & CG \\
Sophie Domingues (2010) & Brain & GSE24265 & GPL570 & 4 & 4 & 3 \\
\hline
\end{tabular}

1 PH: perihematomal area. CW: contralateral white matter. CG: contralateral grey matter 2

3

4 
Table 2 (on next page)

Screening DEGs in ICH by integrated microarray. 
Table 2 Screening DEGs in ICH by integrated microarray.

\begin{tabular}{ll}
\hline DEGs & Gene terms \\
\hline Upregulated & CXCL8, CCL20, C15orf48, CXCL1, VMP1, AGPAT9, G0S2, \\
& CXCL3, PTX3, TFPI2, CYTIP, TNFAIP3, PPBP, HSPA6, \\
& BCL2A1, TREM1, CCL4, IL18RAP, PMAIP1, SERPINE1, \\
& HBD, TGFBI, PNP, SELE, TIMP1, IL6, NCF2, PLAUR, \\
& LAPTM5, TCIRG1, OLR1, HBB, DUSP5, ISG20,S100P, \\
& PROK2, METRNL, CTGF, CD93,HCAR3, ANXA2P2, \\
& C5AR1, TLR2, FGR,MARCO, HMOX1, TUBB6, CYB5R2 \\
& ADM, UPP1, SLC2A3, CD163, BCAT1,CLEC5A, CCL8, \\
& HLA-DRA, TNFRSF12A,ANXA2, S100A11. \\
& NXPH1, PRRT2, SLITRK5, SOWAHA, CNTN3, \\
& CACNA2D3, MUM1L1, ZNRF3.
\end{tabular}




\section{Table 3 (on next page)}

Top 10 of the most significantly enriched GO terms.

GO, Gene Ontology; BP, biological progress; CC, cellular component; MF, molecular function. 


\begin{tabular}{llcl}
\hline \multicolumn{1}{c}{ Pathway ID } & \multicolumn{1}{c}{ Terms } & & Pene count \\
\hline BP & & 16 & $5.41 \mathrm{E}-12$ \\
GO:0006954 & inflammatory response & 8 & $4.33 \mathrm{E}-09$ \\
GO:0030593 & neutrophil chemotaxis & 13 & $3.67 \mathrm{E}-08$ \\
GO:0006955 & immune response & 7 & $2.35 \mathrm{E}-07$ \\
GO:0070098 & chemokine-mediated signaling pathway & 7 & $5.73 \mathrm{E}-06$ \\
GO:0006935 & chemotaxis & 8 & $1.75 \mathrm{E}-05$ \\
GO:0001525 & angiogenesis & 7 & $3.10 \mathrm{E}-05$ \\
GO:0032496 & response to lipopolysaccharide & 4 & $7.02 \mathrm{E}-05$ \\
GO:0090023 & Positive regulation of neutrophil chemotaxis & 5 & $1.36 \mathrm{E}-04$ \\
GO:0071347 & cellular response to interleukin-1 & 4 & $2.00 \mathrm{E}-04$ \\
GO:0045765 & regulation of angiogenesis & & \\
CC & & 19 & $5.27 \mathrm{E}-07$ \\
GO:0005615 & extracellular space & 19 & $6.79 \mathrm{E}-06$ \\
GO:0005576 & extracellular region & 27 & $9.38 \mathrm{E}-04$ \\
GO:0005886 & plasma membrane & 4 & $2.70 \mathrm{E}-03$ \\
GO:0005604 & basement membrane & 13 & $3.43 \mathrm{E}-03$ \\
GO:0005887 & integral component of plasma membrane & 19 & $6.75 \mathrm{E}-03$ \\
GO:0070062 & extracellular exosome & 7 & $1.18 \mathrm{E}-02$ \\
GO:0009986 & cell surface & 3 & $1.60 \mathrm{E}-02$ \\
GO:0031093 & platelet alpha granule lumen & 5 & $2.02 \mathrm{E}-02$ \\
GO:0031012 & extracellular matrix & 3 & $2.25 \mathrm{E}-02$ \\
GO:0030666 & endocytic vesicle membrane & & \\
MF & & 7 & $2.25 \mathrm{E}-02$ \\
GO:0008009 & chemokine activity & 3 & $2.25 \mathrm{E}-02$ \\
GO:0045236 & CXCR chemokine receptor binding & 5 & $2.25 \mathrm{E}-02$ \\
GO:0008083 & growth factor activity & 4 & $2.25 \mathrm{E}-02$ \\
GO:0002020 & protease binding & 5 & $2.25 \mathrm{E}-02$ \\
GO:0004872 & receptor activity & 3 & $2.25 \mathrm{E}-02$ \\
GO:0048306 & calcpium-dependent protein binding & 2 & $2.25 \mathrm{E}-02$ \\
GO:0008329 & signaling pattern recognition receptor activity & 2 & $2.25 \mathrm{E}-02$ \\
GO:0001849 & complement component C1q binding & 5 & $2.25 \mathrm{E}-02$ \\
GO:0005102 & receptor binding & 2 & $2.25 \mathrm{E}-02$ \\
GO:0004859 & phospholipase inhibitor activity & &
\end{tabular}

Table 3. Top 10 of the most significantly enriched GO terms. 
Table 4 (on next page)

Significantly enriched KEGG pathway.

KEGG, Kyoto Encyclopedia of Genes and Genomes. 
1 Table 4. Significantly enriched KEGG pathway.

\begin{tabular}{|c|c|c|c|c|}
\hline Pathway ID & Terms & Gene Count & P-Value & Genes \\
\hline hsa04060 & $\begin{array}{l}\text { Cytokine-cytokine receptor } \\
\text { interaction }\end{array}$ & 10 & $1.59 \mathrm{E}-09$ & $\begin{array}{c}C C L 4, C C L 8, C X C L 8, C C L 20, T N F R S F 12 A \\
\text { IL18RAP, CXCL3, PPBP, CXCL1, IL6 }\end{array}$ \\
\hline hsa05323 & Rheumatoid arthritis & 7 & $3.50 \mathrm{E}-09$ & $\begin{array}{c}\text { TCIRG1, CXCL8, CCL20, TLR2, CXCL1, HLA- } \\
\text { DRA, IL6 }\end{array}$ \\
\hline hsa05134 & Legionellosis & 6 & $9.62 \mathrm{E}-09$ & CXCL8, TLR2, CXCL3, CXCL1, IL6, HSPA6 \\
\hline hsa04062 & Chemokine signaling pathway & 8 & $3.28 \mathrm{E}-08$ & $\begin{array}{c}C C L 4, C C L 8, C X C L 8, C C L 20, F G R, C X C L 3, \\
P P B P, C X C L 1\end{array}$ \\
\hline hsa04145 & Phagosome & 7 & $1.45 \mathrm{E}-07$ & $\begin{array}{c}\text { TCIRG1, OLR1, NCF2, TLR2, TUBB6, HLA-DRA, } \\
M A R C O\end{array}$ \\
\hline hsa05144 & Malaria & 5 & $2.19 \mathrm{E}-07$ & IL6, CXCL8, HBB, SELE, TLR2 \\
\hline hsa04668 & TNF signaling pathway & 6 & $5.37 \mathrm{E}-07$ & TNFAIP3, CCL20, CXCL3, SELE, CXCL1, IL6 \\
\hline hsa05132 & Salmonella infection & 5 & $3.30 \mathrm{E}-06$ & CXCL1, CCL4, IL6, CXCL3, CXCL8 \\
\hline hsa04621 & $\begin{array}{l}\text { NOD-like receptor signaling } \\
\text { pathway }\end{array}$ & 4 & $1.78 \mathrm{E}-05$ & CXCL1, IL6, TNFAIP3, UPP1 \\
\hline hsa05321 & Inflammatory bowel disease (IBD) & 4 & $2.43 \mathrm{E}-05$ & HLA-DRA, IL6, IL18RAP, TLR2, \\
\hline hsa04064 & NF-kappa B signaling pathway & 4 & $1.04 \mathrm{E}-04$ & CCL4, TNFAIP3, BCL2A1, CXCL8 \\
\hline hsa05143 & African trypanosomiasis & 3 & $1.09 \mathrm{E}-04$ & $I L 6, H B B, S E L E$ \\
\hline hsa05146 & Amoebiasis & 4 & $1.36 \mathrm{E}-04$ & CXCL1, IL6, CXCL8, TLR2 \\
\hline hsa04933 & AGE-RAGE signaling pathway in & 4 & $1.52 \mathrm{E}-04$ & IL6, SERPINE1, SELE, CXCL8 \\
\hline
\end{tabular}


diabetic complications

\begin{tabular}{|c|c|c|c|c|}
\hline hsa04066 & HIF-1 signaling pathway & 4 & $1.63 \mathrm{E}-04$ & IL6, SERPINE1, TIMP1, HMOX1 \\
\hline hsa05142 & $\begin{array}{c}\text { Chagas disease (American } \\
\text { trypanosomiasis) }\end{array}$ & 4 & $1.69 \mathrm{E}-04$ & IL6, CXCL8, SERPINE1, TLR2 \\
\hline hsa04620 & $\begin{array}{l}\text { Toll-like receptor signaling } \\
\text { pathway }\end{array}$ & 4 & $1.81 \mathrm{E}-04$ & CCL4, IL6, CXCL8, TLR2 \\
\hline hsa05162 & Measles & 4 & 4.47E-04 & IL6, TNFAIP3, HSPA6, TLR2 \\
\hline hsa05120 & $\begin{array}{l}\text { Epithelial cell signaling in } \\
\text { Helicobacter pylori infection }\end{array}$ & 3 & 7.57E-04 & CXCL1, TCIRG1, CXCL8 \\
\hline hsa05140 & Leishmaniasis & 3 & $8.21 \mathrm{E}-04$ & $H L A-D R A, N C F 2, T L R 2$ \\
\hline hsa05152 & Tuberculosis & 4 & $1.04 \mathrm{E}-03$ & TCIRG1, IL6, HLA-DRA, TLR2 \\
\hline hsa05164 & Influenza A & 4 & $1.06 \mathrm{E}-03$ & HLA-DRA, IL6, HSPA6, CXCL8 \\
\hline hsa04610 & $\begin{array}{c}\text { Complement and coagulation } \\
\text { cascades }\end{array}$ & 3 & $1.15 \mathrm{E}-03$ & C5AR1, PLAUR, SERPINE1 \\
\hline hsa05169 & Epstein-Barr virus infection & 4 & $1.85 \mathrm{E}-03$ & HLA-DRA, FGR, TNFAIP3, HSPA6 \\
\hline hsa05145 & Toxoplasmosis & 3 & $3.57 \mathrm{E}-03$ & HLA-DRA, HSPA6, TLR2 \\
\hline hsa05332 & Graft-versus-host disease & 2 & $4.53 \mathrm{E}-03$ & $H L A-D R A, I L 6$ \\
\hline hsa04672 & $\begin{array}{l}\text { Intestinal immune network for } \operatorname{IgA} \\
\text { production }\end{array}$ & 2 & $6.46 \mathrm{E}-03$ & $H L A-D R A, I L 6$ \\
\hline hsa05161 & Hepatitis B & 3 & $6.47 \mathrm{E}-03$ & IL6, CXCL8, TLR2 \\
\hline hsa05150 & Staphylococcus aureus infection & 2 & $8.12 \mathrm{E}-03$ & $H L A-D R A, C 5 A R 1$ \\
\hline
\end{tabular}

2 KEGG, Kyoto Encyclopedia of Genes and Genomes. 


\section{Table 5 (on next page)}

Table5. Significantly enriched GO of hub genes.

GO, Gene Ontology; BP, biological progress; CC, cellular component; MF, molecular function; 
1 Table5. Significantly enriched GO of hub genes.

\begin{tabular}{|c|c|c|c|c|}
\hline ID & Description & P-Value & Count & Gene Names \\
\hline \multicolumn{5}{|l|}{$\mathbf{B P}$} \\
\hline GO:0009611 & response to wounding & $4.58 \mathrm{E}-11$ & 9 & $\begin{array}{l}\text { CXCL1/ IL6/ CCL20/ SERPINE1/ TLR2/ SELE/ } \\
\text { CCL4/ PLAUR/ CD163. }\end{array}$ \\
\hline GO:0006954 & inflammatory response & $1.45 \mathrm{E}-08$ & 7 & $\begin{array}{l}\text { CXCL1/ IL6/ CCL20/ TLR2/ SELE/ CCL4/ } \\
\text { CD163. }\end{array}$ \\
\hline GO:0006952 & defense response & $6.44 \mathrm{E}-07$ & 7 & $\begin{array}{l}\text { CXCL1/ IL6/ CCL20/ TLR2/ SELE/ CCL4/ } \\
\text { CD163. }\end{array}$ \\
\hline GO:0006935 & chemotaxis & $2.27 \mathrm{E}-06$ & 5 & CXCL1/ IL6/ CCL20/ CCL4/ PLAUR. \\
\hline GO:0006955 & immune response & $2.25 \mathrm{E}-04$ & 4 & CXCL1/ IL6/ CCL20/ TLR2/ CCL4. \\
\hline GO:0007596 & blood coagulation & $6.88 \mathrm{E}-04$ & 5 & IL6/ SERPINE1/ PLAUR. \\
\hline GO:0050817 & coagulation & 0.001396979 & 3 & IL6/ SERPINE1/ PLAUR. \\
\hline GO:0007599 & hemostasis & 0.001957914 & 3 & IL6/ SERPINE1/ PLAUR. \\
\hline GO:0042742 & defense response to bacterium & 0.001957914 & 3 & IL6/ CCL20/ TLR2. \\
\hline GO:0050878 & regulation of body fluid levels & 0.002354575 & 3 & IL6/ SERPINE1/ PLAUR. \\
\hline GO:0032101 & regulation of response to external stimulus & 0.003084628 & 4 & IL6/ SERPINE1/ SELE. \\
\hline GO:0042060 & wound healing & 0.003701403 & 3 & IL6/ SERPINE1/ PLAUR. \\
\hline GO:0032755 & positive regulation of interleukin- 6 production & 0.004681351 & 3 & IL6/ TLR2. \\
\hline GO:0042127 & regulation of cell proliferation & 0.006688038 & 3 & CXCL1/ IL6/ SERPINE1/ TIMP1. \\
\hline GO:0042246 & tissue regeneration & 0.012573365 & 2 & SERPINE1/ PLAUR. \\
\hline GO:0032675 & regulation of interleukin- 6 production & 0.012647695 & 4 & IL6/ TLR2. \\
\hline GO:0006953 & acute-phase response & 0.02109503 & 2 & IL6/ CD163. \\
\hline GO:0030162 & regulation of proteolysis & 0.023703896 & 2 & PLAUR/ TIMP1. \\
\hline GO:0050900 & leukocyte migration & 0.02630658 & 2 & IL6/ SELE. \\
\hline
\end{tabular}




$\begin{array}{lllll}\text { GO:0051091 } & \text { positive regulation of transcription factor activity } & 0.034722761 & 2 & \text { IL6/ TLR2. } \\ \text { GO:0045765 } & \text { regulation of angiogenesis } & 0.037299313 & 2 & \text { IL6/ SERPINE1. } \\ \text { GO:0031099 } & \text { regeneration } & 0.039227714 & 2 & \text { SERPINE1/ PLAUR. } \\ \text { GO:0043388 } & \text { positive regulation of DNA binding } & 0.04115268 & 2 & \text { IL6/ TLR2. } \\ \text { GO:0031349 } & \text { positive regulation of defense response } & 0.044992331 & 2 & \text { IL6/ TLR2. } \\ \text { GO:0050727 } & \text { regulation of inflammatory response } & 0.049454644 & 2 & \text { IL6/ SELE. } \\ \text { CC } & & & \text { CXCL1/ IL6/ CCL20/ SERPINE1/ SELE/ CCL4/ } \\ \text { GO:0009611 } & \text { extracellular region } & 2.86 E-06 & 9 & \text { PLAUR/ CD163/ TIMP1. } \\ \text { GO:0006954 } & \text { extracellular region part } & & \text { CXCL1/ IL6/ CCL20/ SELE/ CCL4/ TIMP1. } \\ \text { GO:0006952 } & \text { extracellular space } & 2.31 E-04 & 6 & \text { CXCL1/ IL6/ CCL20/ SELE/ CCL4. } \\ \text { MF } & & 8.30 E-04 & 5 & \\ \text { GO:0005125 } & \text { cytokine activity } & & \text { CXCL1/ IL6/ CCL20/ CCL4. } \\ \text { GO:0008009 } & \text { chemokine activity } & 2.62 E-04 & 4 & \text { CXCL1/ CCL20/ CCL4. } \\ \text { GO:0042379 } & \text { chemokine receptor binding } & 4.35 E-04 & 3 & \text { CXCL1/ CCL20/ CCL4. } \\ \text { GO:0019899 } & \text { enzyme binding } & 4.94 E-04 & 3 & \text { SERPINE1/ SELE/ PLAUR. }\end{array}$


Table 6(on next page)

Table 6. Significantly enriched KEGG pathway of hub genes.

KEGG, Kyoto Encyclopedia of Genes and Genomes. 
1 Table 6. Significantly enriched KEGG pathway of hub genes.

\begin{tabular}{|c|c|c|c|c|}
\hline Pathway ID & Terms & Gene Count & P-Value & Genes \\
\hline hsa04668 & TNF signaling pathway & 4 & $5.54 \mathrm{E}-08$ & $\begin{array}{c}C X C L 1, C C L 20, I L 6, \\
\text { SELE. }\end{array}$ \\
\hline hsa04066 & HIF-1 signaling pathway & 3 & $6.38 \mathrm{E}-06$ & IL6, SERPINE1, TIMP1. \\
\hline hsa04060 & Cytokine-cytokine receptor interaction & 3 & $9.63 \mathrm{E}-05$ & CXCL1, CCL20, IL6. \\
\hline hsa04621 & NOD-like receptor signaling pathway & 2 & 0.000199993 & CXCL1,IL6. \\
\hline hsa04610 & Complement and coagulation cascades & 2 & 0.000364662 & SERPINE1,PLAUR. \\
\hline hsa04620 & Toll-like receptor signaling pathway & 2 & 0.000671108 & IL6,TLR2. \\
\hline hsa04062 & Chemokine signaling pathway & 2 & 0.00200085 & CXCL1,CCL20. \\
\hline hsa04151 & PI3K-Akt signaling pathway & 2 & 0.006358451 & IL6,TLR2. \\
\hline hsa04672 & Intestinal immune network for $\operatorname{Ig} \mathrm{A}$ production & 1 & 0.016724268 & IL6 \\
\hline hsa04623 & Cytosolic DNA-sensing pathway & 1 & 0.022711959 & IL6 \\
\hline hsa04115 & p53 signaling pathway & 1 & 0.02481751 & SERPINE1 \\
\hline hsa01521 & EGFR tyrosine kinase inhibitor resistance & 1 & 0.029714789 & IL6 \\
\hline hsa04068 & FoxO signaling pathway & 1 & 0.047713858 & IL6 \\
\hline
\end{tabular}

2 KEGG, Kyoto Encyclopedia of Genes and Genomes. 
Table 7 (on next page)

Results of CMap analysis

CMap, Connectivity Map. 
1 Table 7 Results of CMap analysis

\begin{tabular}{llllll}
\hline Rank & CMap name & Mean & $\mathrm{N}$ & Enrichment & P-value \\
\hline 1 & hecogenin & -0.613 & 4 & -0.931 & 0.00002 \\
2 & pralidoxime & -0.529 & 4 & -0.872 & 0.00056 \\
3 & metolazone & -0.282 & 5 & -0.779 & 0.00096 \\
4 & lidocaine & -0.258 & 5 & -0.766 & 0.0013 \\
5 & NU-1025 & -0.579 & 2 & -0.956 & 0.00414 \\
6 & ambroxol & -0.269 & 4 & -0.774 & 0.00527 \\
7 & pipemidic acid & -0.336 & 3 & -0.846 & 0.00735 \\
8 & yohimbic acid & -0.345 & 3 & -0.845 & 0.00747 \\
9 & chlorphenesin & -0.354 & 4 & -0.74 & 0.00899 \\
\hline
\end{tabular}

2 CMap, Connectivity Map. 